\title{
Optical FM System for Measuring Mechanical Shock
}

\author{
L. D. Ballard and W. S. Epstein, \\ Institute for Basic Standards, National Bureau of Standards, Washington, D.C. 20234 \\ E. R. Smith, \\ Harry Diamond Laboratories, U.S. Army Materiel Command, Washington, D.C., 20438 \\ and
}

S. Edelman

Institute for Materials Research, National Bureau of Standards, Washington, D.C. 20234

(August 13, 1969)

\begin{abstract}
A technique is described for calibrating shock accelerometers by measuring the Doppler shift in light frequency produced by the change in velocity of a target. The system employs a quadrature laser interferometer and a single-sideband carrier insertion circuit to distinguish between positive and negative velocities.
\end{abstract}

Key words: Accelerometer; calibration; Doppler; interferometer; laser; shock measuring; singlesideband.

\section{Introduction}

Shock loading may be one of the most destructive or most beneficial environments encountered by mechanical systems. The damage to structures by sudden loads, such as produced by the collision of objects, is familiar. The forming of materials into desired shapes by forging or explosive forming techniques are examples of desirable effects of controlled impact, or shock loading.

There is a demonstrated need to measure shock loads accurately over a wide range of amplitudes and wave forms. The design of structures to withstand such loads and the efficient use of energy for forming materials are only two of the more obvious benefits of a knowledge of shock loadings and their effects.

One of the problems in measureing mechanical shock has been the inability to calibrate shock measuring systems absolutely except by subjecting them to intense, steady state, sinusoidal motion at a few discrete frequencies. Such calibrations are inherently suspect because they are not characteristic of the environment to be measured, and transducers are frequently damaged during such tests. Sinusoidal calibrations of this nature offer the added difficulty that the tests are very time consuming and expensive.

Shock measuring transducers can also be calibrated by measuring lumped response integrated over the total energy spectrum with no attempt to determine response as a function of frequency. An example of this type of calibration is a peak value calibration where the transducer is subjected to a shock input and calibrated as to the peak reading obtained. The calibration comes from either a reference transducer or computed accelerations via measuring velocity changes over finite time intervals. There is a need for a fast, accurate and absolute method for calibrating shock measuring systems under conditions closely simulating those encountered in actual use [1-7]. ${ }^{1}$

\section{Shock Measuring System}

An interferometer system has been devised to provide an absolute calibration facility for measuring shock induced motion. This system produces an electrical signal that is directly proportional to the velocity of the moving element being studied. This signal can be processed, analyzed and compared to the signal from a transducer that is subjected to the same motion. The system has the following advantages: (1) it is a system completely independent of the transducer, (2) it is based on the Doppler shift of light frequency detected by a moving interferometric fringe pattern, a phenomenon that is known to be linear with velocities

\footnotetext{
${ }^{1}$ Figures in brackets indicate the literature references at the end of this paper.
} 
in the range of the mechanical shock spectrum, (3) it is capable of measuring actual shock motion having a wide range of amplitudes and rich in frequency content, and (4) the resulting signal can be rapidly and accurately analyzed by known methods and techniques [8].

\subsection{Theory}

The moving reflector of an optical interferometer is attached to the object whose motion is to be measured. A narrow section of the fringe pattern is viewed by a photodetector; if the interferometer plates are uncoated the photodetector output is [9].

$E_{1}=A+E_{0} \sin [k(\delta+2 \xi)]$

$E_{1}=$ output voltage

$E_{0}=$ signal out, dependent upon light intensity

$A=\mathrm{d}$-c term

$k=2 \pi / \lambda$

$\lambda=$ wavelength of monochromatic light

$\delta=$ average optical separation between interfering

light beams

$\xi=$ displacement of reflector

If we had, instead of eq (1)

$E_{2}=A+E_{0} \sin [\omega t+k(\delta+2 \xi)]$

$\omega=$ carrier frequency

which is a frequency modulated wave [10], the velocity of the reflector could be obtained by demodulation. The output of a demodulator is proportional to the instantaneous frequency deviation and the instantaneous frequency in eq $(2)$ is

$$
f_{1}=\frac{1}{2 \pi} \frac{d}{d t}[\omega t+k \delta+2 k \xi]=\frac{\omega}{2 \pi}+\frac{2 \dot{\xi}}{\lambda}
$$

Therefore, the instantaneous frequency will define what the instantaneous velocity is. Equation (3) could have been obtained by direct application of doppler shift equation [11].

If $E_{1}$ in eq (1) were demodulated directly, or even after up-conversion of the frequencies, the sign of the output would be ambiguous. The problem is therefore to insert a carrier into $E_{1}$ of eq (1) and convert it to $E_{2}$ of eq (2).

\subsection{Description}

The interferometer used in the present work is a commercial model shown schematically in figure 1 . It is a modified Michelson type, illuminated by a singlefrequency He-Ne laser so that long path differences are possible. The moving retroreflector is a corner cube for the usual reasons. The interfering beams are circularly polarized so that by use of a beam splitter and analyzers, followed by two photodetectors, two electrical outputs in phase quadrature are obtained. The original purpose of this arrangement is for fringe counting [12]. The instrument has internal preamplifiers of bandwidth DC to $500 \mathrm{kHz}$; for higher velocity external preamplifiers of bandwidth $20 \mathrm{~Hz}$ to $60 \mathrm{MHz}$ were used.

If one output is described by eq (1), which we rewrite, dropping the constant $A$ and replacing $k(\delta+2 \xi)$

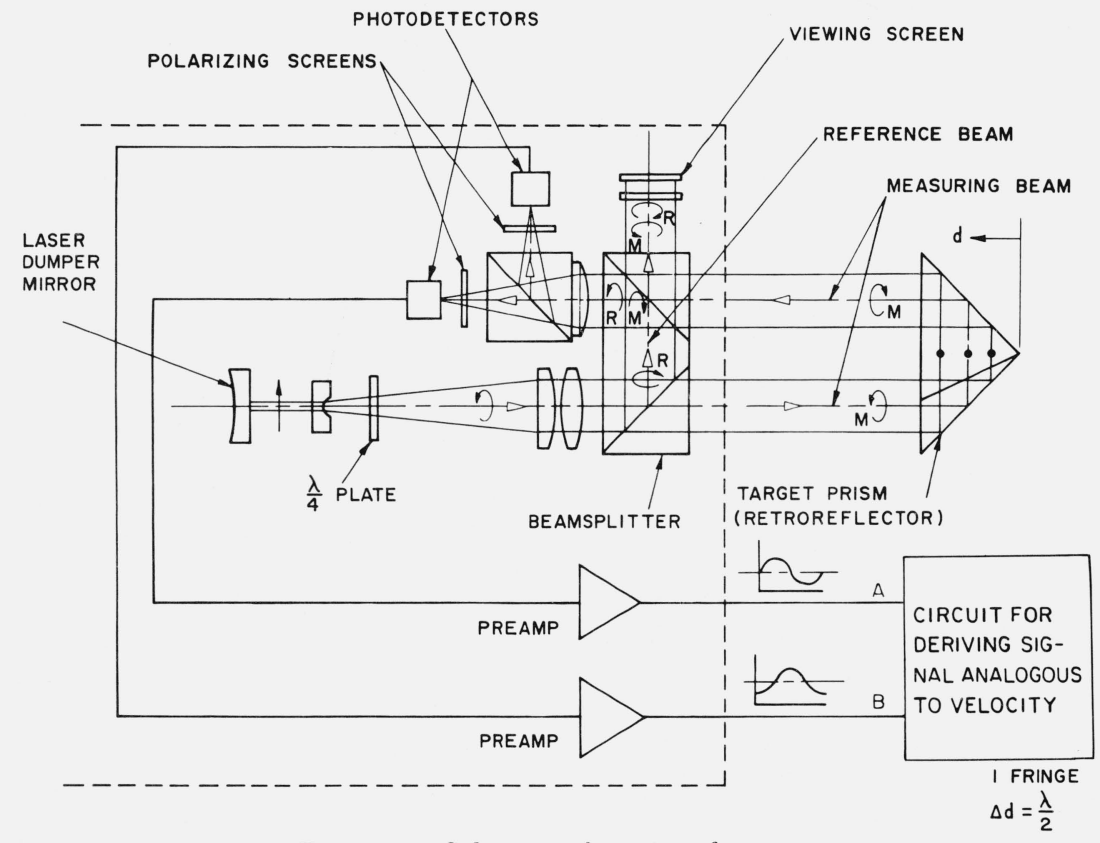

FigURE 1. Schematic, laser interferometer. 
by $x$, as

then the other is

$$
E_{A}=C \sin x
$$

$$
E_{B}=C \cos x
$$

The signal $E_{B}$ is multiplied, in a balanced mixer by the output $D \sin \omega t$ of a local oscillator, giving

$$
C D \sin \omega t \cos x
$$

and $E_{A}$ is multiplied, in another balanced mixer, by $D \cos \omega t$, giving,

$$
C D \cos \omega t \sin k t
$$

the outputs (4) and (5) are added in a suitable circuit to give

$$
C D \sin (\omega t+x)=C D \sin [\omega t+k(\delta+2 \xi)]
$$

which is the frequency-modulated wave of eq (2). As already explained, demodulation of this wave yields an output proportional to the velocity of the reflector.

The above method is similar to the phase-shift method of single-sideband generation [13] with the distinct difference that the signal phase shift is produced optically.

The single-sideband circuit was assembled from off-the-shelf hardware of balanced mixers, quadrature hybrid, FM demodulator and carrier oscillator. The carrier used in this case is $60 \mathrm{MHz}$. The signal is demodulated by a conventional FM discriminator, and the resulting signal can be analyzed by either digital or analog techniques.
The system can be readily calibrated by subjecting the moving element to steady state sinusoidal motion. One technique that has been used involves counting interference fringes over a known time interval. Average and peak velocities can then be computed for the frequency of vibration. Other methods for calibrating the system, such as the Crosby method for determining peak FM swing, could also be used. $[14,15,16]$ Inherent in the system is a possible means of checking the calibration factor during the course of each experiment. The calibration factor can be determined by comparison of the average velocity over a known time interval, during which there are no velocity reversals, with the average discriminator output over the same interval. The former is the displacement, determined from a fringe count, divided by the time interval.

\section{Performance}

To obtain data an accelerometer was attached directly to the face of a corner cube retroreflector. The accelerometer was small enough to fit in a quadrant of the corner cube so as not to interfere with the laser beams. The-moving corner cube of the interferometer was mounted on the table of an electrodynamic shaker. The input signal to the shaker was a single sine pulse $(301 \mathrm{~Hz})$ repeated at a rate of 75.3 Hz. Qualitative comparisons of signals obtained from various subsystems can be made from figures $2 \mathrm{a}, \mathrm{b}$, and c. Figure $2 \mathrm{a}$ shows the signals from the discriminator of the interferometer system and from the accelerometer. Figure $2 \mathrm{a}$ shows the signals from
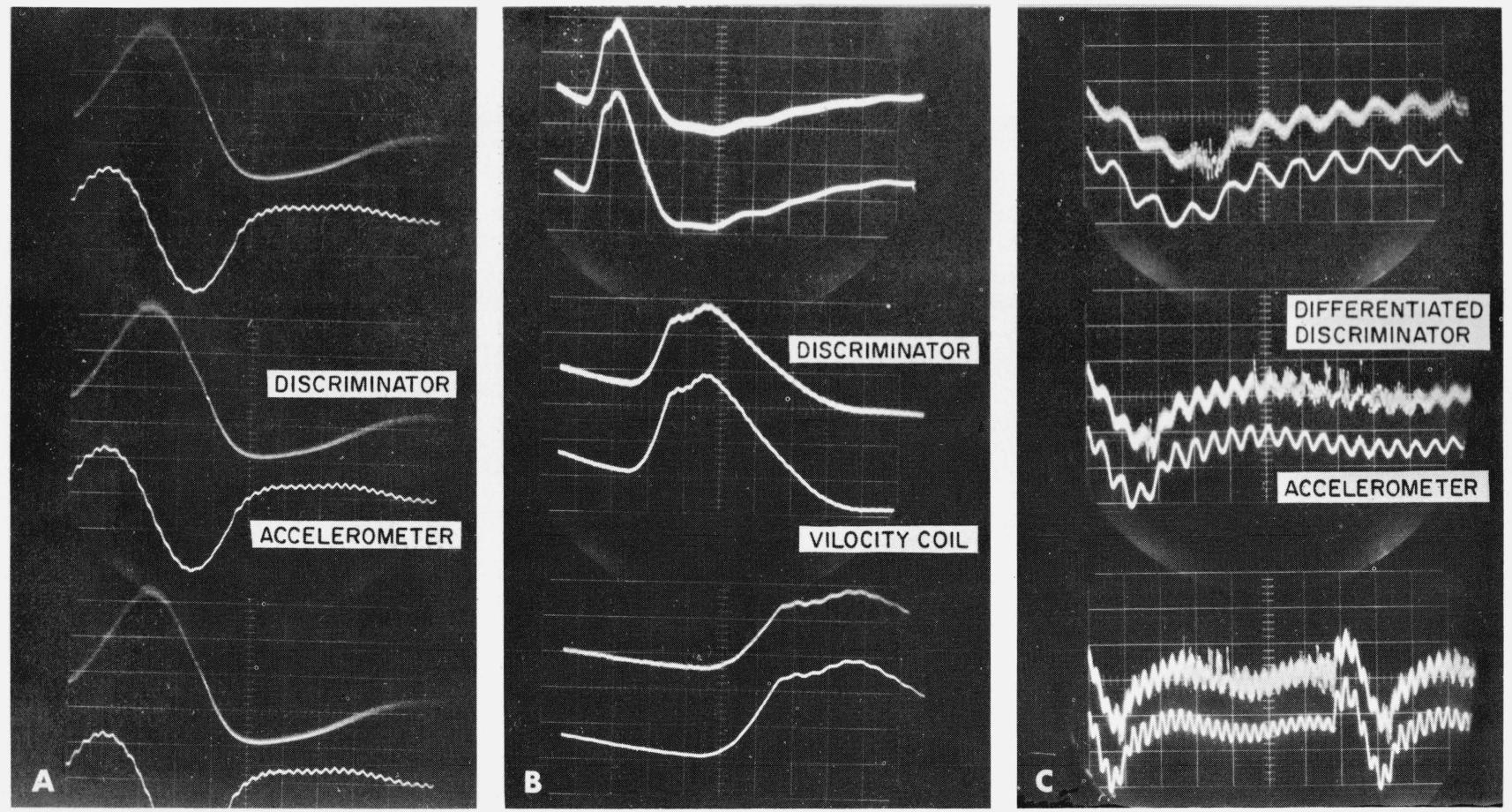

FIGURE 2. Simultaneous signals from shock motion measuring system.

a. Discriminator versus accelerometer, b. discriminator versus velocity coil, $c$. differentiated discriminator versus accelerometer. 
the discriminator of the interferometer system and from the accelerometer. Figure $2 b$ shows the signals from the discriminator and from the velocity coil of the shaker. Figure 2c shows the differentiated output of the discriminator and the output of the accelerometer. The two outputs shown in figure $2 \mathrm{c}$ were fed to a frequency analyzer and the results plotted on an X-Y plotter. The measured peaks of response are shown in table 1 . The calibration factors were arbitrary and the results are only meaningful when compared to each other as shown in the percent deviation column. The differences in-harmonic relationships of the frequencies are due to errors in determining the frequency at a point in a logarithmic sweep.

TABLE 1. Comparison between accelerometer and differentiated discriminator output spectrum due to repetitive sine pulse

\begin{tabular}{c|c|c|c}
\hline \hline Frequency & $\begin{array}{c}\text { Differentiated } \\
\text { discriminator }\end{array}$ & Accelerometer & $\begin{array}{c}\text { b } \\
\text { Accelerometer } \\
\text { output deviation }^{\text {from discrimi- }} \\
\text { nator output }^{\mathrm{a}}\end{array}$ \\
\hline $\mathrm{Hz}$ & Units & Units & Percent \\
76.5 & 4.50 & 4.63 & +3.6 \\
151 & 8.23 & 8.18 & 0.0 \\
225 & 7.76 & 7.75 & +.5 \\
301 & 7.41 & 7.45 & +1.2 \\
376 & 6.46 & 6.72 & +4.6 \\
2104 & 6.02 & 5.75 & -3.8 \\
\hline
\end{tabular}

a Normalized to maximum discriminator amplitude recoreed at $151 \mathrm{~Hz}$

b Amplitude as measured on a X-Y plotter.

\section{Other Applications}

The development of this system was oriented towards motion with large velocities and displacements. If the emphasis is changed from detecting frequency to detecting phase modulation, it is feasible to measure fractional parts of a fringe displacement. [17] This approach is not limited to a laser as an electromagnetic radiation source; any coherent source will do, such as radar.

\section{Summary}

A system has been described that is capable of sensing mechanical shock induced motion and providing a signal that is proportional to the velocity of the motion. The system makes use of the Doppler shift in light frequency, a phenomenon that is known to be linear with amplitude and frequency of motion. The information output is a variable frequency thereby minimizing the linearity requirements for electronic components other than the discriminator. The system can be readily calibrated by use of steady state sinusoidal motion. The information is available in a form that can be analyzed by known methods and techniques.
The authors wish to express their appreciation tor the financial support received from:

\section{U.S. Atomic Energy Commission \\ Sandia Corporation \\ Albuquerque, New Mexico AL-67-421, \\ U.S. Army Missile Command \\ U.S. Army Metrology and Calibration Center \\ Redstone Arsenal, Alabama YP-369,}

Naval Ordnance System Command

Naval Plant Representative

Metrology Engineering Center

Pomona, California $\quad 4355 / \mathrm{Nl}(\mathrm{N} 69-35)$.

\section{References}

[1] Conrad, R. W., and Vigness, Irwin, Calibration of accelerometers by impact techniques, Proc. Inst. Soc. Am. 8, 166 (1953).

[2] Bouche, R. R., The absolute calibration of pickups on a dropball shock machine of the ballistic type, Proc. Inst. Environmental Sci., 115 (1961).

[3] Ramboz, J. D., and Blanchard, C. A., Report No. ESEE08, Endevco 2965 Shock Calibrator, Metrology Engineering Center Naval Plant Representative, Pomona, California; Private Communication.

[4] Burke, R. G., Improved velocity measurement techniques for the shock calibration of accelerometers, Proc. Inst. of Environmental Sci. (1963).

[5] Kelley, R. W., Calibration of shock accelerometers, Proc. 22nd ISA Conf., M18-2-MESTIND-67, Inst. Soc. Am. (1967).

[6] Levy, S., and Kroll, W. D., Response of accelerometers to transient accelerations, J. Res. Nat. Bur. Stand. (U.S.) $\mathbf{4 5}$, No. 4, 303 (1950) RP2138.

[7] Bundy, D. E., The calibration of transducers for use in high intensity shock measurement, Technical Note W.C. 56, Royal Aircraft Establishment, Ministry of Aviation, London W.C. 2, England, March 1964, AD-449463.

[8] IEEE Transactions on Audio and Electroacoustics, AU-15 No. 2, June 1967, Special Issue on Fast Fourier Transform and Its Application to Digital Filtering and Spectral Analysis.

[9] Schmidt, V. A., Edelman, S., Smith, E. R., and Jones, E., Optical calibration of vibration pickups at small amplitudes, J. Acous. Soc. Am. 33, 748 (1961).

[10] Hund, A., in the book Frequency Modulation, Eq. 10, p. 16, McGraw-Hill Book Co., New York (1942).

[11] T. P. Gill, in the book The Doppler Effect, Chapter 5 (Academic Press, New York, 1965).

[12] Rowley, W. R. C., Some aspects of fringe counting in laser interferometers, IEEE, Transactions on Instrumentation and Measurement, Im-15, No. 4, 146 (1966).

[13] Norgaard, D. E., The Phase-Shift Method of Single-Sideband Signal Generation, Proceedings of the IEEE, 44, No. 12, 1718 (1956)

[14] Hund, A., ibid., p. 86.

[15] Smith, D. H., Method for Obtaining Small Mechanical Vibrations of Known Amplitude, Proc. Phy. Soc. (London) 57, 534 (1945).

[16] Ziegler, C. A., Electromechanical Pickup Calibration by the Interferometer Method, J. Acous. Soc. Am. 25, 135 (1953).

[17] von Willisen, F. K., Time resolved photoelectric interferometry of very high phase resolution, Oral Presentation at 1968 Spring Meeting Optical Soc. Am.

(Paper 73C3\&4-291) 